BONPLANDIA 17(1): 83-89. 2008

\title{
EUPATORIUM RUFESCENS Y VERNONIA OLIGACTOIDES (ASTERACEAE), NUEVAS CITAS PARA LA FLORA ARGENTINA
}

\author{
ÁLVARO JOSÉ VEGA ${ }^{1} \&$ MASSIMILIANO DEMATTEIS ${ }^{2}$
}

\begin{abstract}
Summary: Vega, A. J. \& M. Dematteis. 2008. Eupatorium rufescens and Vernonia oligactoides (Asteraceae), new records for the Argentinian flora. Bonplandia 17(1): 83-89. ISSN: 05240476.
\end{abstract}

Eupatorium rufescens Lund ex DC. and Vernonia oligactoides Less. (Asteraceae) are recorded for the first time for Argentina. Both species are described and a key to separate them from related taxa, along with some ecological information on the entities is also presented.

Key words: Eupatorieae, Vernonieae, Compositae, record.

Resumen: Vega, A. J. \& M. Dematteis. 2008. Eupatorium rufescens y Vernonia oligactoides (Asteraceae), nuevas citas para la flora argentina. Bonplandia 17(1): 83-89. ISSN: 0524-0476.

Se mencionan por primera vez para la flora argentina a Eupatorium rufescens Lund ex DC. y Vernonia oligactoides Less. (Asteraceae). Ambas entidades son descriptas y se presenta una clave para diferenciarlas de taxones afines, junto con algunas informaciones ecológicas sobre las entidades.

Palabras clave: Eupatorieae, Vernonieae, Compositae, registro.

\section{Introducción}

En el transcurso de varias campañas de colección efectuadas en la provincia de Misiones se han hallado dos especies de Asteraceae no citadas para Argentina. Una de ellas es Eupatorium rufescens Lund ex DC., entidad conocida hasta ahora solamente para el sur de Brasil, Bolivia y este de Paraguay. Según la clasificación propuesta por Cabrera \& Klein (1989), E. rufescens pertenece a la sect. Laevia Cabrera, que comprende a especies que presentan receptáculo plano, glabro, filarios en 1-2 series, involucro acampanado y
15-25 flores por capítulo. Conforme al esquema sugerido por King \& Robinson (1987), esta entidad está incluida en el género Kaunia R. M. King \& H. Rob., el cual comprende un total de 14 especies exclusivamente sudamericanas. Las únicas especies de este grupo que crecen en Argentina son E. lasiophthalmum Hieron. y E. saltense Hieron., morfológicamente muy diferentes de E. rufescens y restringidas al noroeste del país (Cabrera, 1978).

La otra entidad inédita para nuestro país es Vernonia oligactoides Less., una especie muy vistosa por su follaje cubierto de pubescencia ocrácea. Este taxón pertenece a la sección Lepidaploa subsect. Paniculatae Benth. \&

${ }^{1}$ Facultad de Ciencias Exactas y Naturales y Agrimensura, Universidad Nacional del Nordeste, Corrientes.

${ }^{2}$ Instituto de Botánica del Nordeste, Casilla de Correo 209, 3400, Corrientes. 
Hook., la cual ha sido recientemente segregada al género Vernonanthura H. Rob. (Robinson, 1992). Las especies de este grupo se caracterizan por presentar inflorescencias tirsoideas o corimbosas amplias, filarios pequeños, receptáculo desnudo, corola glabra, rafidios cuadrangulares u oblongos y granos de polen equinolofados, tricolporados, con tectum continuo, micro-perforado, provisto de espinas prominentes (Dematteis, 2006). En Argentina habitan un total de 15 especies de la subsección, las cuales se concentran principalmente en las regiones montañosas del noroeste y en los campos y selvas del litoral, en Misiones y Corrientes (Cabrera, 1944; Cristóbal \& Dematteis, 2003). Las formas biológicas de las especies presentes en Argentina comprenden arbustos, arbolitos y árboles de más de 20 metros de altura, como es el caso de $V$. petiolaris (DC.) H. Rob., una especie selvática endémica del área superior del río Paraná.

El propósito del presente trabajo es dar a conocer la presencia de Eupatorium rufescens y Vernonia oligactoides en Argentina, entidades que no fueron citadas para este país en los trabajos florísticos o catálogos existentes (Cabrera \& al., 1997; Cabrera \& Freire, 1999; Cristóbal \& Dematteis, 2003; Freire \& al., 2006). Ambas especies se describen y se presenta una clave para distinguirlas de taxones afines.

\section{Resultados}

\section{Eupatorium rufescens Lund ex DC.}

De Candolle, A. P., Prodr. 5: 168. 1836; Baker, in Mart., Fl. Bras. 6: 348. 1876; B. L. Rob., Contr. Gray Herb. 61: 67. 1920; Malme, Kungl. Sv. Vet. Akad. Handl. 12(2): 48. 1933. Typus: Rio de Janeiro, $P$. W. Lund s. $n$. (holotypus G-DC).

Eupatorium rufescens var. glabratum Hieron., in Kuntze, Rev. Gen. Plant. 3: 148. 1898. nomen.

Eupatorium rufescens var. glabratum Hieron. ex B. L. Rob., Contr. Gray Herb. 61: 67. 1920; Cabrera \& Vittet, Sellowia 15: 204. 1963; Cabrera \& Klein, Fl. Il. Catar. COMP 4: 602. 1989; Cabrera, Fl. Paraguay 25: 168. 1996. Typus: Bolivia. Cochabamba. Río Tapacari, Alt. 3000 ft., 19-III-
1892, O. Kuntze s. n. (holotypus NY!, foto).

Eupatorium subtriplinerve Sch. Bip. ex Baker, in Mart., Fl. Bras. 6: 348. 1876. nom. nud. pro syn.

Kaunia rufescens (Lund ex DC.) R. M. King \& H. Rob., Phytologia 47(3): 260. 1980.

Iconografía: Cabrera, Fl. Paraguay 25: 169, fig. 64. 1996.

Arbusto erecto, ramoso, de 1,5-3 m alt. Tallos estriados, hojosos hasta la inflorescencia, tomentosos en las partes jóvenes, glabros a la madurez. Hojas membranáceas, opuestas, pecioladas, con pecíolo de 3-6 cm long. Lámina anchamente ovada, crenada o groseramente dentada, acuminada en el ápice, deltoidea y generalmente asimétrica en la base, pentanervada, punteado-glandulosa, glabra en la haz, laxamente pubescente en el envés, principalmente sobre las venas, de 10-15 cm long. x 6-11 cm lat. Capítulos numerosos, reunidos una inflorescencia paniculiforme más o menos amplia. Involucro acampanado, notablemente más corto que las flores, de 4-6 mm alt. x 4-5 mm diám. Filarios laxamente imbricados, dispuestos en 2 series casi similares en longitud, oblongos u oblongolanceolados, agudos, laxamente pubescentes en el dorso, de 3,5-4,2 mm long. Flores 20-25, blanquecinas o blanco-rosadas. Corola de 5-6 $\mathrm{mm}$ long. Aquenios de 2-2,5 mm long., glabrescentes. Papus blanquecino.

Distribución y hábitat: Habita en el interior y bordes de selvas del sur de Brasil (Paraná, Río de Janeiro, Río Grande do Sul, Santa Catarina), sur de Bolivia y este de Paraguay (Concepción, Guairá). En nuestro país ha sido hallada en el extremo norte de Misiones, en las Cataratas del Iguazú, y en el este de esta misma provincia, sobre el río Uruguay cerca de la localidad de Panambí.

Fenología: Florece y fructifica durante los meses de agosto y septiembre.

Material examinado. ARGENTINA. Misiones: Dep. Iguazú, Parque Nacional Iguazú, Cataratas de Iguazú, sendero de observación, selva marginal, 7-VIII-1991, R. Vanni, S. Ferrucci, M. G. López \& S. Chiquizola 2770 (CTES); Parque 
Nacional Iguazú, paredón frente a la Isla San Martín, con neblina permanente, 10-VIII-1995, $R$. Vanni, V. Maruñak \& A. Schinini 3441 (CTES); Dep. Oberá, Ruta prov. 2 (costera), al norte de Panambí, borde de selva, elevación 114 m, 27VIII-2007, H. A. Keller, M. Gartland \& A. Bohren 4271 (CTES).

Material adicional estudiado. BRASIL. Rio Grande do Sul: Santa María, 10-IX-1976, K. Hagelund 10411 (CTES); São Pedro, Montenegro, 7-IX-1949, A. Sehnem 3813 (CTES).

Obs.: Kuntze (1898) cita el nombre de Eupatorium rufescens var. glabratum adjudi- cándole la autoría de la variedad a Hieronymus, quien nunca publicó dicha entidad en forma válida. Posteriormente, Robinson (1920) en un tratamiento sobre las especies de Eupatorieae de Bolivia, cita este mismo nombre pero además describe brevemente la variedad típica y la variedad glabratum y cita el material de herbario determinado por el mismo Hieronymus. Por tal motivo, Cabrera \& Vittet (1963), Cabrera \& Klein (1989) y Cabrera \& al. (1996), le adjudican directamente la autoría de la variedad a Robinson, dado que este autor es quien proporciona una diagnosis y valida el nombre de Hieronymus.

\section{Clave para diferenciar a E. rufescens de especies afines de Argentina}

1. Hojas glabras en ambas caras, lustrosas en la haz.

E. saltense Hieron.

1'. Hojas pubescentes en el envés, principalmente sobre las nervaduras, opacas en la haz.

2. Flores 12-20. Hojas aserradas.

E. lasiophthalmum Hieron.

2’. Flores 20-25. Hojas crenadas o groseramente dentadas.

E. rufescens Lund ex DC.

\section{Vernonia oligactoides Less.}

(Fig. 1)

Lessing, C. F., Linnaea 4: 648. 1831. Typus: Brasil. F. Sellow s. n. (holotypus B, isotypus P!).

Vernonia conyzoides Chodat, Bull. Herb. Boissier ser. 2, 3: 303. 1902. Typus. Paraguay. Dep. Canindeyú. In uliginosis Ipé-hú, Sierra de Maracayú, 9-IX-1898/1899, E. Hassler 5289 (holotypus G!).

Vernonanthura oligactoides (Less.) H. Rob., Phytologia 73(2): 72. 1992.

Vernonia sorocabae Sch. Bip. ex Baker, in Mart., Fl. Bras. 6(2): 58. 1873, nom. nud. pro syn.
Sufrútice de 1-2 m alt., con tallos erectos, ramas gradualmente más cortas hacia la parte superior, ocráceo-tomentosas, densamente hojosas hasta el ápice, con entrenudos de 2-4 cm de largo. Hojas coriáceas, sésiles, obovadas, agudas u obtusas en el ápice y atenuadas en su mitad inferior, enteras o ligeramente crenadas, glabras y lustrosas en la cara superior, densamente ocráceo-tomentosas en la inferior, con nervaduras prominentes, de 6$10 \mathrm{~cm}$ long. x 2-4 cm lat. Capítulos muy numerosos, sésiles o brevísimamente pedunculados, formando glomérulos densos, que se sitúan en los ápices de ramas cortas 


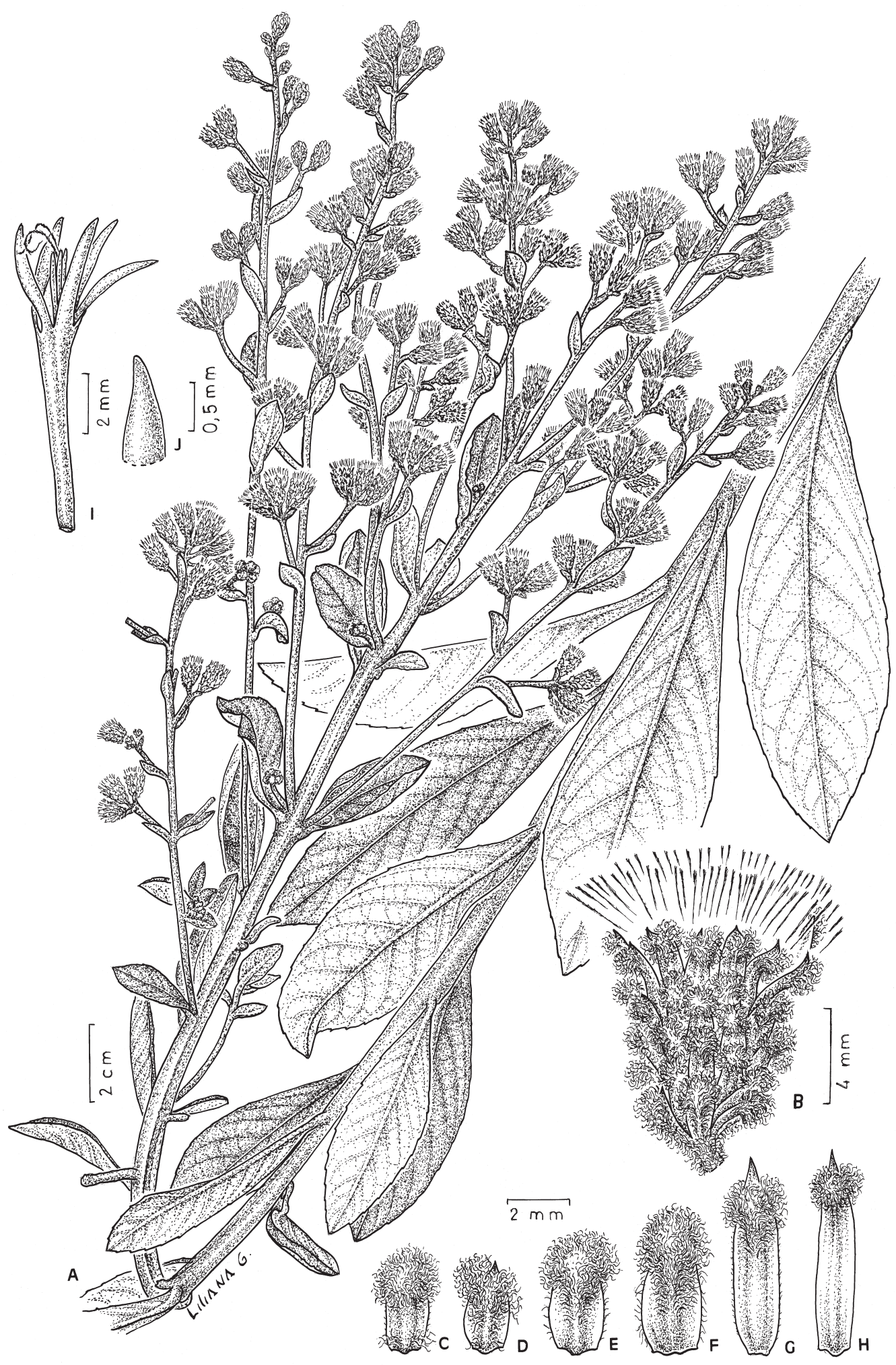

Fig. 1. Vernonia oligactoides Less. A: planta. B: capítulo. C-D: filarios externos. E-F: filarios intermedios. G-H: filarios internos. I: corola con anteras y estilo. J: ápice del lóbulo de la corola (A-J, Hatschbach \& al. 76946). 
que nacen de las axilas de las hojas superiores. Involucro turbinado, de $10-12 \mathrm{~mm}$ alt. $\mathrm{x}$ 5-8 mm diám. Filarios dispuestos en 8-9 series, coriáceos, los exteriores ovados, cortos, los interiores oblongo-lanceolados, todos cortamente mucronados en el ápice y lanosos en la parte superior del dorso. Flores 20-25, liláceas o rojizas. Corola de 10-11 mm long., lóbulos lanceolados de 2,5-3,6 mm long. Anteras de 3-3,6 mm long. Estilo de 11,5-13,5 mm long., con ramas de 2,5-3,5 $\mathrm{mm}$ long. Aquenios costados, pubescentes de 3,5-4 mm long. Papus blanco, serie externa paleácea, de 0,6-0,6 mm long., serie interna setácea, de 7-9 mm long. 2n=34.

Distribución y hábitat: Es una especie característica de las serranías, campos y cerrados del sur de Brasil (Mato Grosso do Sul, Paraná, Santa Catarina, São Paulo) y este de Paraguay (Alto Paraná, Amambay, Canindeyú). En Argentina crece en una pequeña área con suelo pedregoso cercana a Bernardo de Irigoyen, caracterizada por numerosas especies campestres acompañadas por Araucaria angustifolia (Bertol.) Kuntze.

Fenología: Florece en verano y otoño, desde febrero hasta mayo.

Material estudiado: ARGENTINA. Misiones: Dep. General Manuel Belgrano, Ruta prov. 17, Campinas de América, cementerio, 2-VII-
2006, H. Keller 3547 (CTES); Dep. General Manuel Belgrano, Campinas de Américo, 26¹6’28"S-534'ㄷㅇ"W, 720 m s.m., 19-IV2007, M. Dematteis \& al. 2611 (CTES).

Material adicional estudiado: BRASIL. Mato Grosso do Sul: Mun. Ponta Porã, Fazenda Itamarati, Rod. MS-164, 9-III-2004, G. Hatschbach \& al. 76946 (CTES). Paraná: Mun. Jaguariaíva, Parque Estadual do Cerrado, 11-X-1999, L. Von Linsingen \& Sonehara 113 (CTES). São Paulo. Itararé, margen esquerda do Rio Verde, 8-IV-1995, P. Aoki s. n. (FUEL, UEC). PARAguAY. Alto Paraná: Pirá Pytá, $12 \mathrm{~km}$ NE de Hernandarias, Ea. Santa Elena, 250 m s.m., $25^{\circ} 17^{\prime}$ S, 543' W, A. Schinini \& al. 28164 (CTES). Amambay: Colonia Estrella, 50 km de Pedro Juan Caballero, 9-XII-1997, M. Dematteis \& A. Schinini 862 (CTES); Estancia 5 Hermanos, camino a la colonia Pirity, 20-IV1996, N. Soria 7550 (CTES); Alrededores de

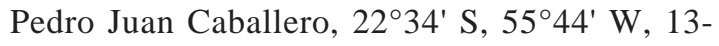
III-1996, A. Schinini \& al. 30436 (CTES); Chiriguelo, ca. $550 \mathrm{~m}$ s.m., 6-XII-1997, M. Dematteis \& A. Schinini 854 (CTES); Pedro Juan Caballero, camino a Cerro Corá, Ruta 5,

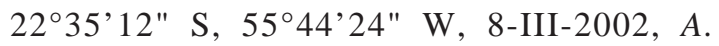
Schinini \& al. 36022 (CTES).

Obs.: Por su aspecto, $V$. oligactoides puede ser confundida con $V$. cuneifolia Gardner y $V$. mucronulata Less., pero se diferencia fácilmente de éstas por la pubescencia de tallos y hojas.

\section{Clave para diferenciar a $V$. oligactoides de especies afines de Argentina}

1. Arbustos de hasta $3 \mathrm{~m}$ alt. Tallos muy ramificados. Sin la presencia de xilopodio.

2. Capítulos con 6-11 flores.

2'. Capítulos con 11-15 flores.

V. nitidula Less.

V. squamulosa Hook. \& Arn.

1'. Sufrútices o hierbas perennes de hasta $2 \mathrm{~m}$ alt. Tallos ramificados o sencillos. Con xilopodio.

2. Hojas obovadas u elíptico-ovadas.

3. Hojas obovadas, de base atenuada y ápice agudo a obtuso. Filarios lanosos en la superficie dorsal.

V. oligactoides Less. 
3'. Hojas elípticas u ovadas, de base redondeada a semi-acorazonada y ápice agudo. Filarios glabros en la superficie dorsal.

V. chamaedrys Less.

2'. Hojas lineares, oblanceoladas o lanceoladas.

3. Hojas estrechamente lineares, con margen revoluto.

4. Involucro acampanado, de 8-10 mm long. Filarios muy apretados.

V. nudiflora Less.

4'. Involucro turbinado, de 8-12 mm long. Filarios laxamente imbricados.

V. cupularis Chodat

3'. Hojas oblanceoladas o lanceoladas, con margen más o menos revoluto.

4. Hojas tomentosas en el envés.

V. chaquensis Cabrera

4'. Hojas glabras en el envés.

5. Haz de las hojas lisa. Capítulos con 10-12 flores.

V. lucida Less.

5'. Haz de la hojas áspera. Capítulos con 18-20 flores.

V. schulziana Cabrera

\section{Agradecimientos}

La ilustración fue preparada por Mirta Liliana Gómez del Instituto de Botánica del Nordeste. Este trabajo se realizó mediante el apoyo financiero del Consejo Nacional de Investigaciones Científicas y Tecnológicas (CONICET), la Myndel Botanica Foundation y la Secretaría General de Ciencia y Técnica de la Universidad Nacional del Nordeste (UNNE).

\section{Bibliografía}

CABRERA, A. L. 1944. Vernonieas Argentinas (Compositae). Darwiniana 6: 265-379.

—_. 1978. Compositae. Tribu Eupatorieae. Fl. Prov. Jujuy 10: 56-156.

—— \& N. ViTTET. 1963. Compositae catharinensis. II. Eupatorieae. Sellowia 15: 149-258.

__ \& R. M. KLEIN. 1989. Compostas. 4. Tribo: Eupatorieae. Fl. Il. Catarin. 1(COMP): 413-760.

\& S. E. FREIRE. 1999. Asteraceae.
Eupatorium. In F. O. Zuloaga \& O. Morrone (eds.), Catálogo de las Plantas Vasculares de la República Argentina II. Monogr. Syst. Bot. Missouri Bot. Gard. 74: 170-186.

_—, — \& M. M. CERANA. 1997. Asteraceae VIII. Tribu II. Eupatorieae. Fl. Fanerog. Argentina 47: 3-98. Museo Botánico, Córdoba.

— W. C. HOLMES \& S. MCDANIEL. 1996. Compositae III. Fl. Paraguay. 25: 1-349.

CRISTÓBAL, C. L. \& M. DEMATTEIS. 2003. Asteraceae, Tribu I. Vernonieae. Fl. Fanerog. Argentina 83: 3-53. Museo Botánico, Córdoba.

DEMATTEIS, M. 2006. Vernonanthura warmingiana (Asteraceae: Vernonieae), a new species from Brazil. Brittonia 58(2): 182-188.

FREIRE, S. E., E. URTUBEY, G. SANCHO, N. D. BAYÓN, L. KATINAS, D. G. GUTiÉRreZ, D. A. GIULIANO, A. A. SÁENZ, L. IHARLEGUI \& G. DELUCCHI. 2006. Inventario de la biodiversidad vegetal de la provincia de Misiones: Asteraceae. Darwiniana 44: 375-452.

KING, R. M. \& H. ROBINSON. 1987. The genera of the Eupatorieae (Asteraceae). Monogr. Syst. Bot. Missouri Bot. Gard. 22: 1-581.

KUNTZE, O. 1898. Compositae. Revis. Gen. Pl. 
A. J. Vega \& M. Dematteis, Eupatorium rufescens y Vernonia oligactoides, nuevas citas para Argentina

3: 127-185.

ROBINSON, B. L. 1920. The Eupatoriums of Bolivia. Contr. Gray Herb. 3(61): 30-80.
ROBINSON, H. 1992. A new genus Vernonanthura (Vernonieae: Asteraceae). Phytologia 73: 65-76.

Original recibido el 19 de mayo de 2008; aceptado el 25 de junio de 2008. 
BONPLANDIA 17(1): 83-89. 2008 